\title{
The Effect of Pelvic Adjustment on the Stability of Elderly Men
}

\author{
Giduck PARK, PhD ${ }^{1)}$, Sung-BUm Ju, PhD ${ }^{2)}$, HyUn JeONG JANG, MSc, PT ${ }^{3)}$ \\ 1) Department of Health \& Fitness Management, Namseoul University \\ ${ }^{2)}$ Department of Health \& Physical Education, Kochi University: \\ 2-5-1, Akebono-cho, Kochi, 780-8520 Japan. TEL: +81 88-844-8391, FAX: +81 88-844-8391, \\ E-mail:accent-ju@daum.net \\ ${ }^{3)}$ Graduate School of Daejeon University
}

\begin{abstract}
Purpose] The purpose of this study was to examine the effect of pelvic adjustment using the Gonstead technique on the stability of elderly men. [Subjects] In this study, 20 elderly men were divided into a pelvic adjustment group (PAG, $\mathrm{n}=10$ ) and a control group $(\mathrm{CG}, \mathrm{n}=10)$. [Methods] Using a Balance Performance Monitor (SMS Health care, England), the sway area, sway path length, and sway maximum (max) velocity of the center of the body were measured to assess the stability of the subjects. [Results] Changes in the variables of stability were analyzed, and based on the results, the PAG showed significant improvement in all items (the sway area, sway path length and sway max velocity of the center of the body) after the intervention of the pelvic adjustment compared to before while the CG did not show any significant improvement. [Conclusion] Pelvic adjustment using the Gonstead technique can be used to improve the stability of elderly men.

Key words: Pelvic adjustment, Equilibrium, Elderly men
\end{abstract}

(This article was submitted Jul. 18, 2011, and was accepted Aug. 4, 2011)

\section{INTRODUCTION}

In elderly persons, abnormal curvature appears due to degenerative changes in the regions between the spinal segments and the strength of the muscles around the pelvis and the spine ${ }^{1)}$. Furthermore, sacroiliac joint malfunction, pelvic misalignments, and differences in the lengths of the legs resulting from aging occur, increasing the risk of falls and reducing the ability to perform activities of daily living and gait ability ${ }^{2)}$. It has been reported that $10-25 \%$ of elderly persons who experience falls, fall due to an insufficient sense of balance and an abnormal gait ${ }^{3)}$. Moreover, for the body to maintain balance, along with normal inputs of the sense of balance, support from the musculoskeletal system, which is a biodynamic aspect of the body, is deemed necessary ${ }^{4}$.

The pelvis supports the abdomen, connects the spine with the lower extremities, and is involved in weight support by the spine and the lower extremities during standing to make the trunk stable and maintain upright positions, to support smooth movements of the upper extremities ${ }^{5)}$. Furthermore, the pelvis should be in a neutral position for the individual to maintain proper balance and appropriately adjust the upper and lower extremities in dynamic positions, to support the ability to perform activities of daily living and gait ${ }^{6)}$. For pelvic adjustment, the Gonstead technique is considered to have the best effects, as it provides excellent pelvic analysis and applies vector force using shear and torsional forces, to recover the normal functioning of moving segments and eliminate dysneuria $^{7,8)}$.

There is evidence that in elderly persons, pelvic misalignment and changes in the position of the pelvis appear in the musculoskeletal system as a result of aging. Consequently, overall stability decreases and the risk of falls increases. Therefore, in this study we used the Gonstead technique, as it has excellent pelvic adjustment effects in elderly persons, and examined the changes in stability in order to elucidate its efficacy and clinical possibilities as a pelvic adjustment therapy.

\section{SUBJECTS AND METHODS}

The subjects of this study were 20 elderly men aged around 70 years, living in D metropolitan city in Korea. They were selected through medical examinations that excluded those who were at risk of complications in pelvic adjustment. The purpose of this study and the entire procedure of the experiment were explained to the subjects, and voluntary consent was received from the subjects before they participated in the experiment. The subjects' lower extremities showed at least $10 \mathrm{~mm}$ differences, and when the pelvic adjustment was administered, the subjects' pelvic subluxation was examined.

The pelvic adjustment group (PAG, $n=10)$ that participated in this study was aged $71.00 \pm 4.64$ years (mean $\pm \mathrm{SD}$ ), and had a height of $168.9 \mathrm{~cm} \pm 3.95 \mathrm{~cm}$ and a weight of $62.30 \mathrm{~kg} \pm 8.94 \mathrm{~kg}$; and the control group (CG, 
Table 1. The comparison of sway parameters in the each group (unit: Nm)

\begin{tabular}{cccc}
\hline Group & Item & Pre $($ Mean \pm SD) & Post (Mean \pm SD) \\
\hline \multirow{3}{*}{ PAG } & sway area* & $207.2 \pm 188.4$ & $55.4 \pm 20.7$ \\
& sway path length* & $244.0 \pm 39.1$ & $144.7 \pm 40.4$ \\
& sway max velocity* & $45.2 \pm 8.9$ & $29.0 \pm 9.0$ \\
\multirow{2}{*}{ CG } & sway area & $184.2 \pm 123.6$ & $125.0 \pm 58.9$ \\
& sway path length & $225.4 \pm 42.3$ & $207.6 \pm 49.1$ \\
& sway max velocity & $52.0 \pm 15.8$ & $42.8 \pm 9.8$ \\
\hline
\end{tabular}

*p<0.05, PAG; Pelvic adjustment group, CG; Control group.

$\mathrm{n}=10)$ was aged $70.30 \pm 3.49$ years, and had a height of $166.80 \mathrm{~cm} \pm 3.35 \mathrm{~cm}$ and a weight of $60.90 \mathrm{~kg} \pm 4.62 \mathrm{~kg}$. There were no statistically significant differences between these characteristics of the two groups ( $p>0.05)$; thus, the two groups were considered to be homogeneous.

In this study, pelvic adjustment therapy was administered by first massaging the buttocks and the lumbar area to relax the surrounding muscles for around 10 minutes and then the Gonstead technique was used. To administer the Gonstead technique, the therapist first aligned the lower extremities of the subjects in the prone position and checked the lengths of the two lower extremities. Then, the subjects lay down in a lateral recumbent position and the therapist implemented the technique through the application of brief, repeated force to the pelvis delivered posterior-inferiorly in the anterior-superior direction and pulling the pelvis down anterior-superiorly in the posterior-inferior direction. The pelvic adjustment was performed 18 times in total, 3 times a week for 6 weeks, and the correction was performed by a skilled therapist with at least 10 years of clinical experience. The control group did not receive any treatment.

To measure the subjects' equilibrium, the sway area, sway path length, and sway maximum (max) velocity of the center of the body were measured using a Balance Performance Monitor (SMS Health care, England) ${ }^{9)}$ before the intervention and at six weeks of the intervention. Comparison of the sway parameters before and after the intervention was conducted with the paired t-test using the SPSS 13.0 Windows program. A significance level, $\alpha$, of 0.05 was chosen.

\section{RESULTS}

The PAG showed significant improvements in all items, the sway area, sway path length and sway max velocity of the center of the body, after the intervention of the pelvic adjustment compared to before $(\mathrm{p}<0.05)$, while the $\mathrm{CG}$ did not show any significant improvement (Table 1).

\section{DISCUSSION}

The pelvis is a structure between the hip joint and the lumbosacral region that is attached to many muscles which adjust the movements of the hip joint and the lumbosacral region. Therefore, the position of the pelvis is the most important factor in determining the body's sagittal alignment and postures ${ }^{10}$. In the case of elderly persons with high risks of falls, the imbalance between the two sides of the pelvis and the spine brings about imbalance in the lower extremities which eventually manifest in imbalance of the trunk ${ }^{11)}$.

Trunk stability can be defined as a stable static condition in which the head of the body is located accurately above the sacrum and the pelvis. If this stability has problems, abnormal curvature will occur in a part of the body, imbalance will arise, and pain in the lumbar area or the back may occur in some cases ${ }^{12)}$. If the trunk is located anterior to the original position, the load imposed on the lumbar spine and the two hip joint axes will increase, and it has been reported that in conditions of imbalance in the sagittal plane, energy consumption in activities increases and stability decreases ${ }^{13)}$. In this respect, given that pelvic displacement resulting from aging of elderly persons is a major factor that reduces stability, we administered pelvic adjustment using the Gonstead technique in an attempt to enhance stability.

The changes in the body sway parameters after the pelvic adjustment was performed using the Gonstead technique were analyzed. The PAG showed significant improvements in all items, the sway area, sway path length and sway max velocity of the center of the body, after the intervention of the pelvic adjustment as compared to before $(\mathrm{p}<0.05)$, while the $\mathrm{CG}$ did not show any significant improvement.

The Gonstead technique is considered to be able to reduce pain by reducing the stress created by abnormal positions of tissues surrounding the joints and correcting minor abnormal positions by subluxation and displacement when the positional relationships between the joints are abnormal $^{14)}$.

In studies of the effect of pelvic adjustment involving the administration of the Gonstead technique, it was shown that the sacral posterior-inferior-leftward (PI-L) displacement in misaligned pelvises were first corrected, and after eight weeks of performing the correction technique, the sacral PI-L recovered its normal position and the iliac internal rotating displacement showed good responses ${ }^{6,8)}$. In a study of pelvic adjustment and equilibrium, pelvic adjustment using the Gonstead technique was performed on children with differences in the length of their lower extremities exceeding $10 \mathrm{~mm}$. The sway area was reduced showing the effect of pelvic adjustment on stability ${ }^{15}$. In a study where sacroiliac joint mobilization and a lumbar stabilizing exercises were conducted as interventions for healthy adults, decreases in sway area and sway length were reported ${ }^{16)}$. Considering the abovementioned studies and 
the results of the present study, and we think given that the pelvic imbalance resulting from aging is a cause of reduced stability, we think that the application of the Gonstead technique corrected the neutral position of the pelvis relieving the imbalance between the pelvis and the spine resulting in compensatory actions in the spinal joints which tended to maintain the balance that occurs in stable pelvic support planes. Therefore, pelvic adjustment using the Gonstead technique for the elderly men enhanced their stability, suggesting it is a method which can be used to improve the stability of elderly men.

\section{REFERENCES}

1) Gauchard GC, Gangloff $P$, Jeanel $C$, et al.: Physical activity improves gaze and posture control in the elderly. Neurosci Res, 2003, 45: 409-417.

2) Lee CS, Lee CK, Kim YT, et al.: Dynamic imbalance of the spine in degeneration flat back. Spine, 2001, 26: 2029-2035.

3) Morse J: Nursing research on patient falls in health care institutions. Annu Rev Nurs Res, 1993, 11: 299-316.

4) Horak FB, Shuperta CL, Mirkaa A: Components of postural dyscontrol in the elderly. Neurobiol Aging, 1989, 10: 727-738.

5) Kapandji IA: Physiology of the joints. Philadelphia: Churchill Livingstone, 2007.

6) Bae SS, Kim TY, Jung HA, et al.: Discussion of the pelvis kinematics. Korean Soc Phys Ther, 1999, 11: 93-102.
7) Plaugher G: Textbook of clinical chiropractic: a specific biomechanical approach. Baltimore: Lippincott Williams \& Wilkins, 1993.

8) Cooperstein R: Gonstead Chiropractic Technique (GCT). J Chiropr Med, 2003, 2: 16-24.

9) Sackley CM, Baguley BI: Visual feedback after stroke with the balance performance monitor: Two single-case studies. Clin Rehabil, 1993, 7: 189195.

10) Vaz G, Roussouly P, Berthonnaud E, et al.: Sagittal morphology and equilibrium of pelvis and spine. Eur Spine J, 2002, 11: 80-87.

11) Beattie P, Lsaacson K, Riddle D L: Validity of derived measurements of leg length differences obtained by use of a tape measure. Phys Ther, 1990, 70: 150-157.

12) Stokes IA: Three dimensional terminology of spinal deformity. Spine, 1994, 19: 236-248

13) Lazennec JY, Ramare S, Arafati R, et al.: Sagittal alignment in lumbosacral fusion: relations between radiological paramenters and pain. Eur Spine J, 2000, 9: 47-55

14) Cailliet R: Low back pain syndrome. Philadelphia: F.A. Davis Company, 1998.

15) Park GD: The effect of Pelvic Manipulation to primary school Balance, Flexibility, and LLI. Journal of Physical Growth and Motor Development, 13: $13-22$.

16) Gong WT: The effects of sacroiliac joint mobilization and lumbopelvic stabilizing exercise on the equilibrium ability. PT. MD. Dissertation, Daggu University, Daggu, 2005. 